\title{
A Formal Framework for High Level Synthesis
}

\author{
Thomas Kropf*, Klaus Schneider ${ }^{*}$ and Ramayya Kumar ${ }^{* *}$
}

\author{
*Institut für Rechnerentwurf und Fehlertoleranz (Prof. D. Schmid) \\ Universität Karlsruhe, Kaiserstr. 12, 76128 Karlsruhe, Germany \\ **FZI (Prof. D. Schmid), Haid-und-Neu-Str.10-14, 76131 Karlsruhe, Germany \\ email: \{kropf, schneide\}@ informatik.uni-karlsruhe.de, kumar@fzi.de \\ WWW: http://goethe.ira.uka.de/hvg/
}

\begin{abstract}
In this paper, we propose a new approach to formal synthesis which focuses on the generation of verification-friendly circuits. Starting from a high-level implementation description, which may result from the application of usual scheduling and allocation algorithms, hardware is automatically synthesized. The target architecture is based on handshake processes, modules which communicate by a simple synchronizing handshake protocol. The circuits result from the application of only a few basic operations like synchronization, sequential execution or iteration of base handshake processes. Each process is guided by an abstract theorem that is used to derive proof obligations, to be justified after synthesis. Automation has been achieved to the extend that only those "relevant" proof obligations remain to be proven manually, e.g. theorems for data-dependent loops and lemmata about the used data types. The process-oriented implementation language is enriched by loop invariants. If those are given prior to the synthesis process and the underlying data types are only Booleans, i.e. finite-length bitvectors, then the complete synthesis and verification process runs automatically.
\end{abstract}

\section{Introduction}

As high-level synthesis [1] has reached the stage of industrial applications, it has turned out that the implicit assumption, that the generated structures just implement the behavioral specification, does not always hold. Software faults, inevitable in complex design tools, hinder the use of synthesized hardware, especially in safety critical applications, and reduce the cost advantages offered by high-level design tools.

Verifying the design tools by software verification techniques fails as a potential remedy to this situation due to the complexity of the algorithms to be checked. Automatically verifying the synthesized hardware by post verification techniques is possible when using model checking techniques [2]. However, this approach is limited to only small and medium sized circuits. It already fails if a couple of 32 bit registers are used in the data path. More complex circuits may be verified by proof-based approaches [3]. However, these techniques are interactive in general and require a good understanding of mathematical logic and the functioning of the target hardware. Large designs are verifiable if they are hierarchically composed. Obviously, automati- 
cally synthesized hardware is hard to tackle using these approaches, since it is synthesized "flat" and its functioning is, especially if optimized, not always understandable to the human hardware verifier.

In this paper, we propose an approach which makes direct use of high-level synthesis algorithms. The high-level implementation description, resulting from scheduling and allocation is mapped onto a target architecture based on modules which communicate by a simple synchronizing handshake protocol, called handshake processes [4]. Handshake processes represent basic operations of the flow graph, resulting from the previous synthesis steps. The processes are composed to get a circuit, satisfying the specification. During the composition process the circuit is verified against the highlevel specification in order to check the correctness of scheduling and allocation, which have been performed outside the formal framework. The verification process results in proof obligations, which may not be verified automatically. Usually this comprises lemmata describing properties of the used data types. For data-dependent loops, the implementation description language includes loop invariants. Stating these before the synthesis process is started, only data type related proof goals remain to be verified manually. However, this part is also automated if only bitvectors of finitelength are used [5].

This first approach may result in sub-optimal synthesized circuits with regard to area or speed. However, this is not a fundamental drawback of our approach since many of the well-known optimization techniques may be used afterwards. In general, optimization algorithms are easier to verify, e.g. optimizing transformations used in retiming may be checked for their behavior-preserving properties [6]. Moreover, safety critical applications may lead to the acceptance of a certain hardware overhead in favor of correctness, similar to the additional hardware which is accepted for enhanced testability.

The outline of the paper is as follows. In the next section, our approach is compared to related work. After introducing the formal framework, notation and the used logic, the synthesis approach is presented. There, we first present the basic "protocol" of the handshake processes and then show how base and composed processes may be synthesized, using an HDL. The paper ends with experimental results and conclusions.

\section{Related Work}

In contrast to post-hoc verification, formal synthesis aims at deriving correct implementations from specifications. Most existing approaches are based on refinement techniques, where a formal specification is successively transformed into hardware implementations. The best known approach is based on the use of the LAMBDA system [7]. Hardware is specified in higher-order logic and synthesized by decomposing the specification into smaller pieces, which can be mapped onto a set of connected modules representing implementations. Although supported by a graphical interface, the synthesis has to be performed completely manually as well as the proof that the resulting structure is behaviorally equivalent to the specification. Hence these designs require a thorough understanding of the used logic and the proof rules to be able to perform the necessary interactive theorem proving. Thus, the overall design methodol- 
ogy is completely different form classical high-level synthesis and many optimization techniques developed in this area cannot be applied.

The formal synthesis of controllers from temporal logic specifications or timing diagrams has been presented in [8]. Due to the underlying propositional logic, these approaches cannot be extended to data path synthesis.

The approaches aiming at the synthesis of self-timed systems [9] are related to our approach with regard to a similar target structure, namely communicating modules. However, we use a synchronous handshake protocol and use higher-order logics as the underlying formal system instead of process algebras. Moreover, we explicitly provide a framework for coping with data dependent loops.

\section{Basics}

In the following, we use higher order logic as used in the HOL theorem proving environment [10]. In contrast to classical first-order logic, quantification is possible also over functions and predicates. The logic is based on typed lambda calculus, i.e. every term $t$ has a unique type $t y$, written as $t: t y$. We use the usual boolean connectives like $\neg, \wedge, \vee$, or $\rightarrow$ and the symbol $\lambda$ for lambda abstraction.

The HOL system provides a set of basic types like ind, the set of individuals, bool, the set of booleans and num, the natural numbers. More complex types can be defined using type operators, which take types and produce new ones. Given two types $t y_{1}$ and $t y_{2}$, the binary type operator for functions $\rightarrow$ gives the type of all functions from $t y_{1}$ to $t y_{2}$, i.e. $t y_{1} \rightarrow t y_{2}$. The pair operator \# forms the cartesian product type of two types $t y_{1} \# t y_{2}$. Another concept, used in the following, is type polymorphism. There are type variables' $t y_{v a r}$, that can be instantiated with an arbitrary type. In the HOL system, complex types may be defined. Proofs are usually performed interactively in the HOL system. However tactics may be defined to automate certain proof tasks. More details can be found in [10]. Proving theorems in the HOL system is usually performed interactively and in "backward" proof style. The goal to be proved is put on top of a goal stack. Repeated rule applications splits the goal into smaller proof goals until axioms are reached and the respective subgoal is removed form the stack. If the goal stack has been completely emptied, the original goal has been proved. Sets of rule applications can be abbreviated as tactics such that partial automation can be achieved.

In the following, modules are represented as predicates and structures of modules as conjunctions of predicates as used in many hardware verification approaches [11]. We use a discrete linear time modelled by natural numbers num and wires are functions from time to the respective data type, e.g. $x:$ num $\rightarrow$ bool.

Since temporal relationships play an important role for the communication of handshake processes, we use higher-order variants of temporal operators [12, 13, 14].

Specifications of digital circuits can often be described conveniently by the "when" operator [15]. In this paper we use a slightly different semantics and formalization of the when operator which is as follows. The expression $(x \text { when } f)^{t_{0}}$ becomes true, if $x$ becomes true for the first time after $t_{0}$, at which $f$ becomes true. In figure 3-1, a timing diagram for $x$ and $f$ is given, which makes ( $x$ when $f$ ) true at time instant $t_{0}$. 


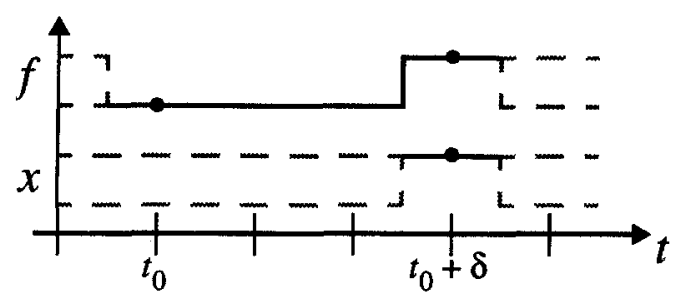

Fig. 3-1. Timing diagram of the when operator

The operator is formally defined in equation 3-1.

$$
(x \text { when } f)^{t_{0}}:=\forall \delta .\left(\forall t .(t<\delta) \rightarrow \neg f^{\left(t_{0}+t\right)}\right) \wedge f^{\left(t_{0}+\delta\right)} \rightarrow x^{\left(t_{0}+\delta\right)}
$$

For the approach, presented in this paper, another definition of the when operator is used (equation 3-2). Intuitively, the when operator can be formalized using a "ready" signal $f$, a "termination" signal $x$ and an invariant $J$ of a data-dependent loop. The invariant must hold at the beginning of the loop $\left(J^{t_{0}}\right)$. If the loop does not terminate $\left(\neg f^{\left(t+t_{0}\right)}\right)$ the invariant must also hold at the next loop iteration. Otherwise, the termination signal $x$ must become true in the next time instance. This order of events is visualized by figure $3-2$.

$$
\begin{aligned}
& (x \text { when } f)^{t}:= \\
& \exists J \cdot J^{t_{0} \wedge} \\
& \quad\left(\begin{array}{l}
\left.\forall t \cdot \neg f^{\left(t_{0}+t\right)} \wedge J^{\left(t_{0}+t\right)} \rightarrow J^{\left(t_{0}+t+1\right)}\right) \wedge \\
\left.\forall \delta \cdot f^{\left(t_{0}+\delta\right)} \wedge J^{\left(t_{0}+\delta\right)} \rightarrow x^{\left(t_{0}+\delta\right)}\right)
\end{array}\right.
\end{aligned}
$$

Fig. 3-2. Visualization of the invariant formalization

Note that the above definitions lead to a "weak when", i.e. it is not required that the signal $f$ ever becomes true. Using the weak version of the operator for specifications leads to partial correctness results, i.e. the hardware gives the right result at the right time, but only if it terminates. In circuits, whose termination is not obvious, e.g. when using data-dependent loops, then an additional termination condition $\exists t . f^{t_{0}}$ has to be shown. ${ }^{1}$ It has been shown previously that, provided that the invariant $J$ is given, proofs of theorems, which contain the temporal operators may be automated [14]. Proof tactics to perform this task are available for the HOL90 theorem proving environment. 


\section{Handshake Circuits}

The main idea underlying this approach is based on the observation that if it is possible to implement modules with a uniform and consistent input/output behavior, then module composition to perform complex computations is almost trivial and the verification of the overall behavior is considerably eased. In most cases the desired correctness theorem, i.e. that the composed structure behaves as specified, may be derived fully automatically.

The interface of a module is as given in figure 4-1. Note that in the following, all modules are described using only one input inp and one output out. However, inp and out are of polymorphic type so that bundles of signals may be easily used as type instances.

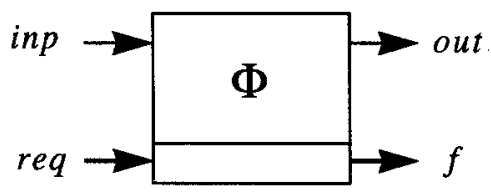

Fig. 4-1. General handshake circuit

Besides input inp and output out two further signals are used: the request input req indicates that a new input value can be read for the next computation and the finish output $f$ denotes the end of a computation cycle. $\Phi$ represents the specification of the module function, i.e. the behavior to be eventually verified. More formally, the module function is expressed by a formula $\Phi$ (inp, out, $t_{1}, t_{2}$ ), where the starting time $t_{1}$ and finish time $t_{2}$ is included. These time instants serve as reference time points for the input and output value and may be also used for formalizing restrictions on the duration of a computation cycle. A typical example is given in equation 4-1 where the sum of $i n p_{1}$ and $i n p_{2}$ is computed, needing 3 time units.

$$
\Phi\left(\text { inp } p_{1}, \text { inp } p_{2}, o u t, t_{1}, t_{2}\right)=\left(o u t^{t_{2}}=i n p_{1}^{t_{1}}+i n p_{2}^{t_{1}}\right) \wedge\left(t_{2}-t_{1}=3\right)
$$

The timing behavior of the module is formalized in terms of the handshake signals $f$ and $r e g$ as stated in equation 4-2. The first implication denotes that provided that no computation is active at time $t_{0}\left(f^{f_{0}}\right)$, then the outputs are stored until a new task is requested (store mode). The second implication states, that after a request for a computation req, the result, computed by $\Phi$, is available at out when $f$ is true again.

$$
\begin{aligned}
& \text { PROTOCOL }(\text { req, in, } f, \text { out }, \Phi):=f^{0} \wedge \\
& \forall t_{0} \cdot\left(f^{t_{0}} \rightarrow\left[\left(\lambda t .\left(\text { out }^{t}=\text { out } t^{t_{0}}\right) \wedge\left(f^{t}=f^{t_{0}}\right)\right) \text { until req }\right]^{t_{0}}\right) \wedge \\
& \left(f^{t_{0}} \rightarrow\left(\left(\lambda t_{1} \cdot\left[\lambda t_{2} \cdot \Phi\left(\text { inp }, \text { out } t_{1}, t_{2}\right) \text { when } f\right]^{\left(t_{1}+1\right)}\right) \text { when req }\right)^{t_{0}}\right)
\end{aligned}
$$

1. It is easily possible to define a strong when when using the weak version as follows: $(x \text { when } f)^{i}:=(x \text { when } f)^{t} \wedge \neg\left((\lambda t . \text { F) when } f)^{t}\right.$ 


\section{Verifying Handshake Circuits}

In this section a high-level implementation description is presented. It is the result of the scheduling and allocation step of high-level synthesis and its semantics is given directly in terms of handshake circuits.

\subsection{Notation}

The language distinguishes two levels of abstraction. For creating base modules, there are constructs for synchronous RT-level descriptions using a clock. For compound modules, process composition constructs are provided as given in table 5-1.

\begin{tabular}{|c|c|c|}
\hline Level & construct & description \\
\hline \multirow{6}{*}{$\begin{array}{l}\text { clock } \\
\text { level }\end{array}$} & $=$ & combinational assignment \\
\hline & $\leftarrow$ & synchronous assignment \\
\hline & T & clock parallel action \\
\hline & op & $\begin{array}{l}\text { arbitrary (combinational) arithmetic or } \\
\text { logic operation }\end{array}$ \\
\hline & bop & $\begin{array}{l}\text { (combinational) boolean operation } \\
\text { resulting in a signal of type bool }\end{array}$ \\
\hline & const & $\begin{array}{l}\text { arbitrary constants (bits, natural } \\
\text { numbers) }\end{array}$ \\
\hline \multirow{3}{*}{$\begin{array}{l}\text { proces } \\
\text { level }\end{array}$} & i & sequential composition \\
\hline & & parallel composition \\
\hline & $:=$ & assignment \\
\hline
\end{tabular}

Table 5-1: Base constructs

Variables in capital letters denote bitvectors, i.e. out $=\left(\right.$ out $_{1}$, out $_{2}, \ldots$, out $\left._{n}\right)$. Sets of registers are abbreviated using an arrow like $\overrightarrow{\mathrm{Out}}=\left(\mathrm{Out}_{1}, \mathrm{Out}_{2}, \ldots, \mathrm{Out}_{q}\right)$.

All processes are defined as follows.

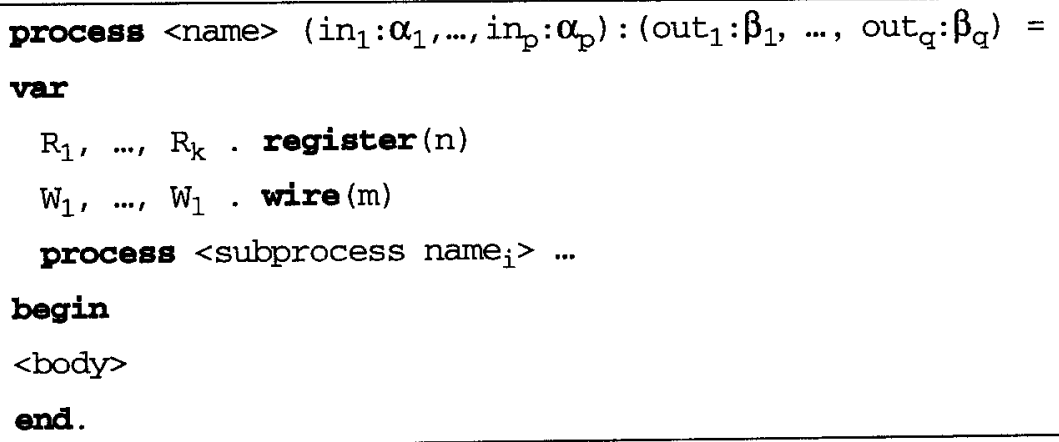


Each process has a name <name>, a set of inputs $i n_{i}$ of type $\alpha_{i}$, resulting in $q$ outputs typed $\beta_{i}$. The types $\alpha_{i}$ and $\beta$ are bitvectors of finite length. The process may declare local registers $R_{i}$ of bitwidth $n$ and wires $W_{i}$ of bit width $m$. The <body> depends on the type of the process. There are basic processes, which are either sequential loops or combinational circuits, and composed processes. The latter are nested loops, sequences or synchronized parallel processes.

A key point of this approach is its ability to cope with arbitrary hierarchical structures. This is possible by guaranteeing that composed modules have the same input/ output behavior as the base modules, i.e. a uniform interface scheme has to be used. In the following base processes, nested loop processes as well as sequential and parallel composition are treated.

\subsection{Base Processes}

A base process usually consists of a data dependent loop and is defined as follows.

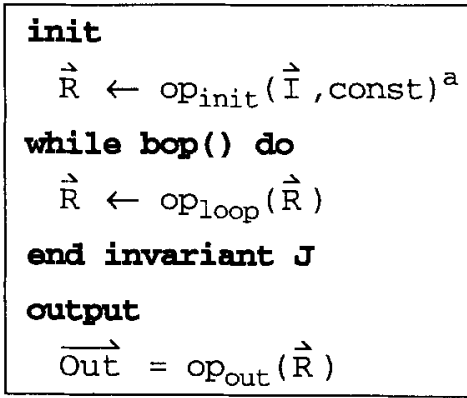

a. abbreviation for

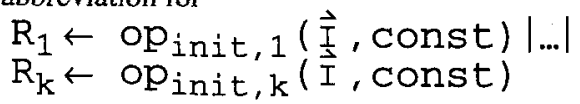

The invariant $J$ is necessary for the automated verification. Currently it is a statement in higher-order logic ${ }^{1}$, using the process signals as arguments. Naturally, a similar construct is also available for REPEAT - UNTIL loops.

The corresponding hardware structure of the module is given in figure 5-1.

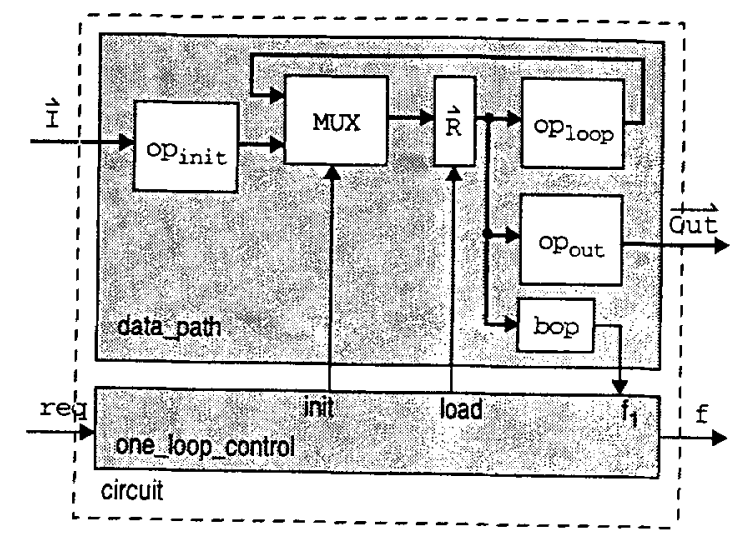

Fig. 5-1. Base handshake process 
The controller is realized according to the state transition graph (STG) of figure 5-2 and hence is the same for every realization of a while loop.

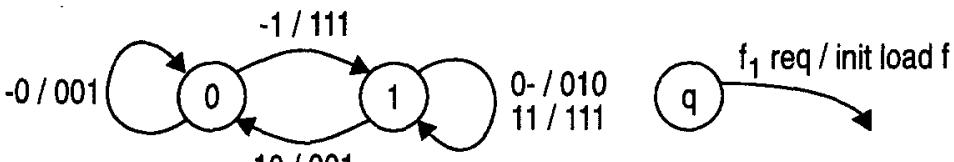

$10 / 001$

Fig. 5-2. STG of one_loop_control

A simple combinational module results from the previous case by omitting the while loop, leading to the simplified structure of figure 5-3. The registers are necessary for maintaining the overall handshake behavior and may be omitted if the input signal remains stable during the whole computation.

$$
\begin{aligned}
& \text { init } \\
& \vec{R} \leftarrow o p_{\text {init }}(\vec{I}, \text { const }) \\
& \text { output } \\
& \overrightarrow{\text { out }}=\text { op out }_{\text {ou }}(\vec{R})
\end{aligned}
$$

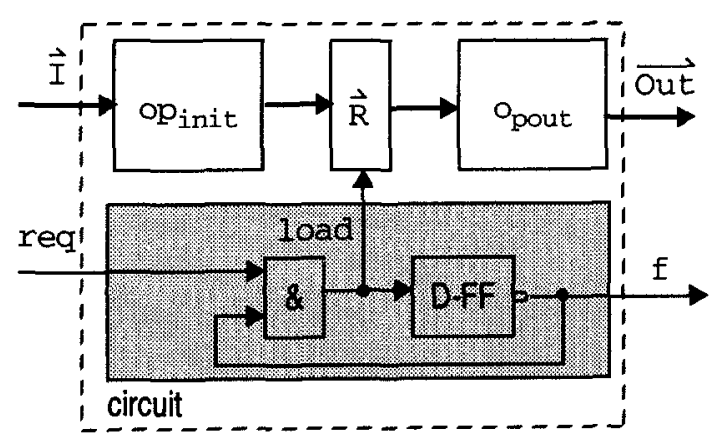

Fig. 5-3. Combinational base handshake process

The register $\vec{R}$ and the controller one_loop_control of figure 5-3 are necessary to store the results of $o_{\text {pout }}\left(o_{\text {init }}(\vec{I})\right)$ to achieve a behavior which conforms to equation 4 2.

A top level formalization of the circuit structure of figure 5-1 in higher-order logic is shown in equation 5-1.

$$
\begin{aligned}
\operatorname{circuit}(\text { req, } \vec{I}, \overrightarrow{\text { out }}, \mathrm{f}):= & \\
\quad \exists \text { init load } \mathrm{f}_{1} \text {. } & \text { data_path }\left(\overrightarrow{\mathrm{I}}, \text { init, load, } \mathrm{f}_{1}, \overrightarrow{\text { out }}\right) \wedge \\
& \text { one_loop_control }\left(\text { req, init, load, } \mathrm{f}_{1}, \mathrm{f}\right)
\end{aligned}
$$

1. Currently the syntax of the HOL90 theorem proving environment has to be used for invariants. 
To verify the module behavior, it has to be shown that the implementation (equation 51) implies a behavioral specification (equation 4-2). This comprises the conformance to the handshake protocol and the computing function $\Phi$. Since the handshake circuits have been synthesized verification-friendly, the timing behavior is automatically verified using appropriate theorems [4]. The remaining proof goal is shown in equation 52.

$$
\mathrm{f}^{t_{0}}, \operatorname{req}^{t_{0}}-\left[\lambda t_{1} . \Phi\left(\overrightarrow{\mathrm{I}}, \overrightarrow{\text { Out }}, t_{0}, t_{1}\right) \text { when } \mathrm{f}\right]^{t_{0}+1}
$$

The remaining proof may be further simplified, if the invariant based on the when definition of equation 3-2 is used. However, the invariant has to be provided manually either only now or already previously in the implementation description.

In the following, a GCD process is defined to illustrate the definition of base handshake processes. The overall process is written down as follows.

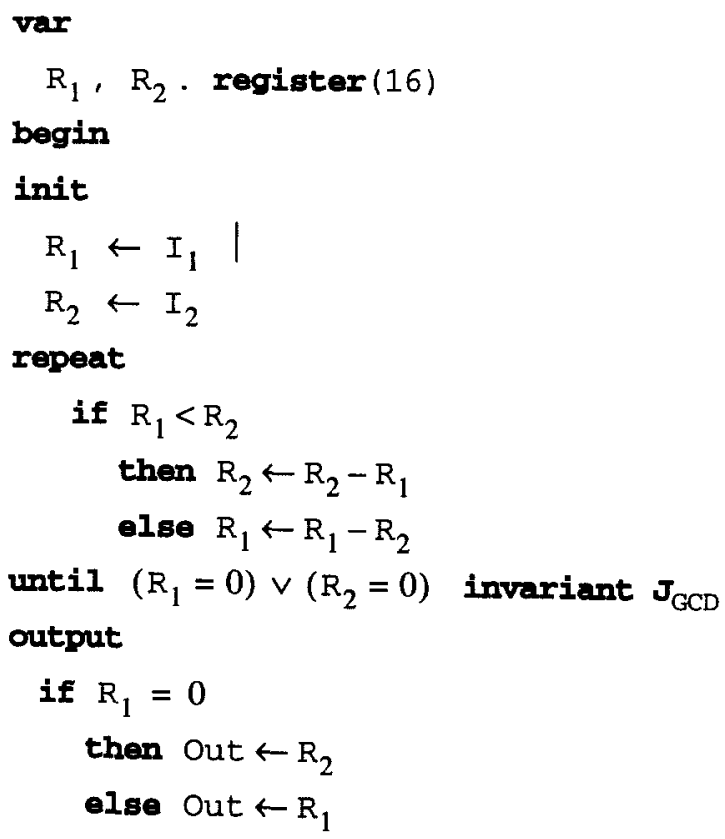

end.

The specification $\Phi$ to be verified is as follows.

$$
\Phi\left(\left[\mathrm{I}_{1}, \mathrm{I}_{2}\right] \text {, Out, } t_{1}, t_{2}\right):=\left(\mathrm{Out}^{t_{2}}=\operatorname{GCD}\left(\mathrm{I}_{1}^{t_{1}}, \mathrm{I}_{2}^{t_{1}}\right)\right)
$$

Based on a usual definition of the Greatest Common Divisor, $G C D$, the invariant 
$\mathbf{J}_{G C D}$ of equation 5-4 is used. Basically it states that the iterative subtraction performed in the registers of the GCD process do not affect the GCD to be computed.

$$
\forall t, t>t_{0} \cdot\left(G C D\left(\mathrm{R}_{1}^{(t+1)}, \mathrm{R}_{2}^{(t+1)}\right)=G C D\left(\mathrm{R}_{1}^{t}, \mathrm{R}_{2}^{t}\right)\right)
$$

\subsection{Compound Processes}

\subsubsection{Nested Loops}

A handshake process may be used to construct a nested data dependent loop. It is called "nested", since most basic handshake processes also contain a data-dependent loop. It thus has a similar function as ${ }^{0} p_{100 p}$ in figure 5-1 but at a higher level of abstraction.

Given a handshake process ${ }_{1}$, which may be defined e.g. as presented in section 5.2.1, nested loops are specified as follows.

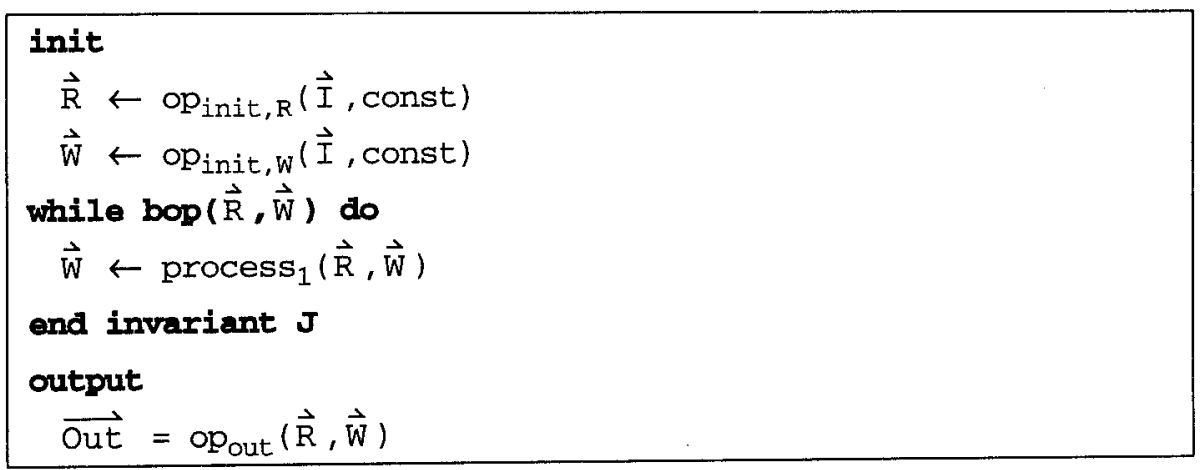

The realization of the nested loop using process 1 is given in figure 5-1.

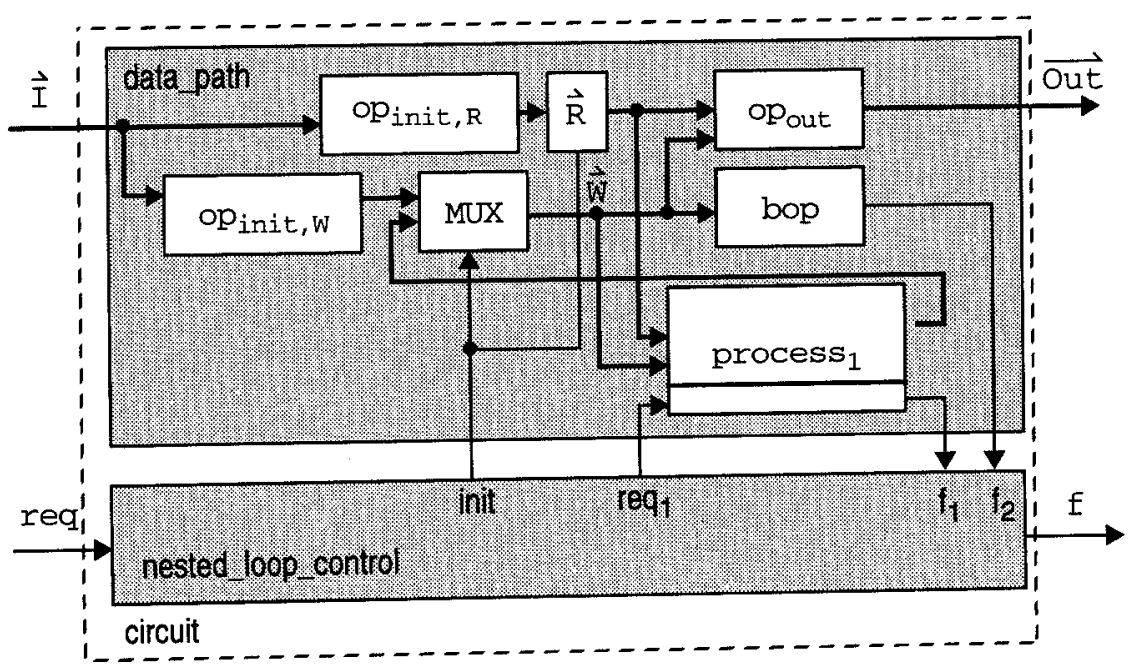

Fig. 5-4. Nested loop handshake process 
The STG of the controller nested_loop_control is given in figure 5-2. Similar to the controller of figure 5-2, it has a wait state and a computation state. The controller starts in the wait state and reaches after a request signal the computation state. The inner loop is started by signal req $_{1}$. Its termination is signalled to the controller by $f_{1}$ and the termination of the outer loop, checked by bop is communicated via $f_{2}$.

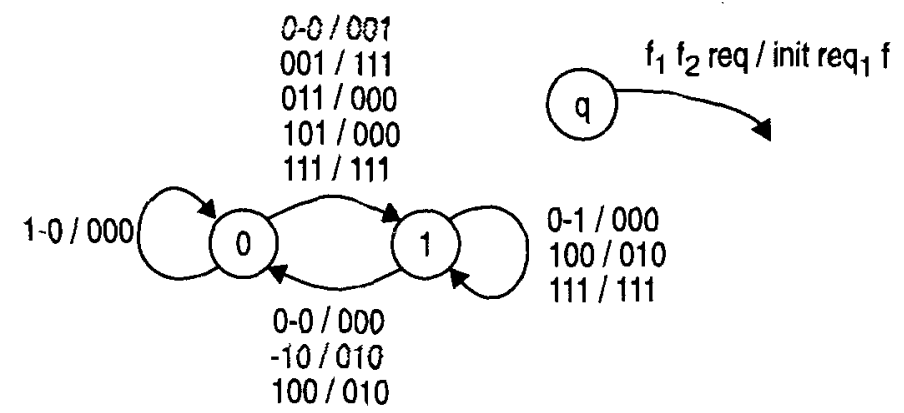

Fig. 5-5. STG of nested_loop_control

As in section 5.2.3, it is sufficient to show, that the following hoids.

$$
\forall t_{0} \cdot \mathrm{f}^{t_{0}} \wedge \operatorname{req}^{t_{0}} \rightarrow\left[\lambda t_{1} . \Phi\left(\overrightarrow{\mathrm{I}}, \overrightarrow{\text { out }}, t_{0}, t_{1}\right) \text { when } \mathrm{f}\right]^{t_{0}+1}
$$

Having proved equation 5-5, the overall behavior PROTOCOL (req, $\vec{I}, \overrightarrow{\text { out }}, f, \Phi$ ) may be derived by a preproven lemma [4] if the controller nested_loop_control is used. The remaining proof goals are similar to those of section 5.2.3 and omitted here.

\subsubsection{Parallel Composition}

The parallel composition or synchronization of two processes is stated as follows.

$\overrightarrow{\mathrm{out}}_{1} \leftarrow \operatorname{process}_{1}\left(\vec{I}_{1}, \overrightarrow{\mathrm{C}}_{1}\right)$,
$\overrightarrow{\mathrm{out}}_{2} \leftarrow \operatorname{process}_{1}\left(\overrightarrow{\mathrm{I}}_{2}, \overrightarrow{\mathrm{C}}_{2}\right)$

The corresponding hardware structure is given in figure 5-6.

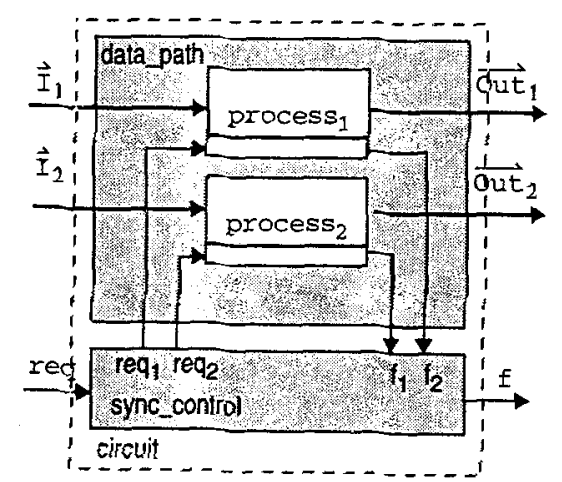

Fig. 5-6. Synchronization of two handshake processes 
The controller sync_control essentially is a simple combinational circuit, where $f$ results from the conjunction of $f_{1}$ and $f_{2}$ and req $_{i}$ from the conjunction of req and f.

The verification of the overall behavior is easy, since a preproven lemma [4] is also available here, stating that, provided that both processes work as intended, then both results are available at $\overrightarrow{\mathrm{out}}_{1}$ and $\overrightarrow{\mathrm{Out}}_{2}$, if $f$ indicates the termination of the composed process.

\subsubsection{Sequential Composition}

The sequential composition of two processes is stated as follows.

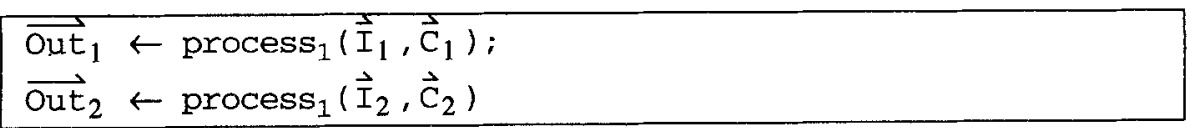

The corresponding hardware structure is given in figure 5-6.

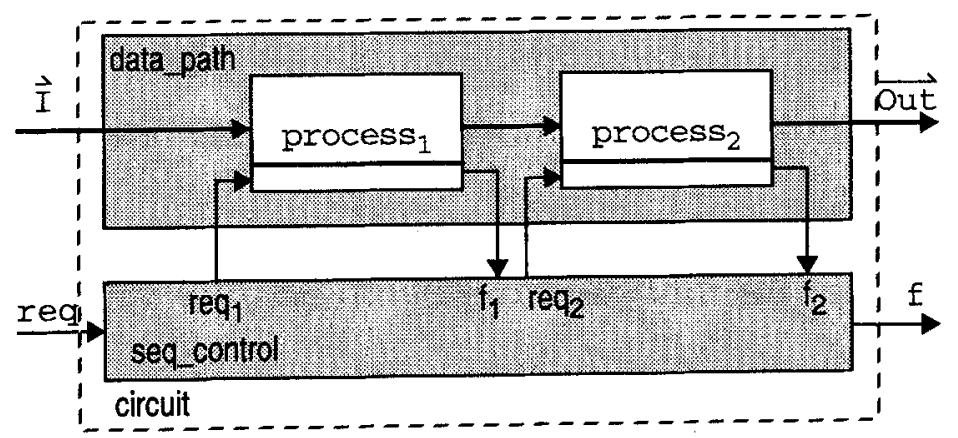

Fig. 5-7. Sequentialization of two handshake processes

The controller seq_control has three states. Its STG is given in figure 5-2. Wait or computation status of both processes are indicated by $W$ and $C$, i.e. the state $C W$ denotes, that the first process is computing a result and the second waits to be served.

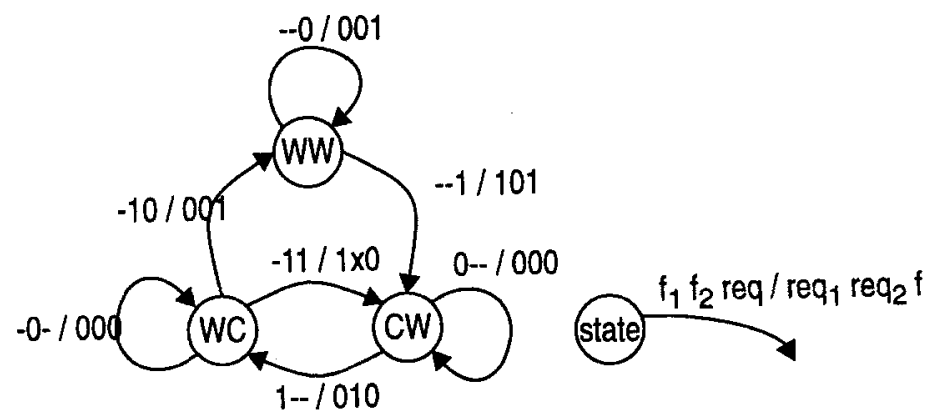

Fig. 5-8. STG of seq_control

Also here a preproven lemma [4] is used to prove, that the whole process provides as a result the functional composition of both processes, if it has terminated. 


\section{Example}

In the following, we will verify a synthesized handshake circuit, which realizes the computation of the euclidean metric, defined in equation 6-1.

$$
\Phi\left(I_{1}, I_{2} \text {, Out, } t_{1}, t_{2}\right)=\left(\text { out }^{t_{2}}=\sqrt{\left(I_{1}^{t_{1}}\right)^{2}+\left(I_{2}^{t_{1}}\right)^{2}}\right)
$$

The overall process is as follows, where Mult, Add and Sqrt are previously defined processes. For computing the square root, e.g. Heron's algorithms based on the following iteration may be used.

$$
x_{n+1}=\frac{1}{2}\left(\frac{a}{x_{n}}+x_{n}\right)
$$

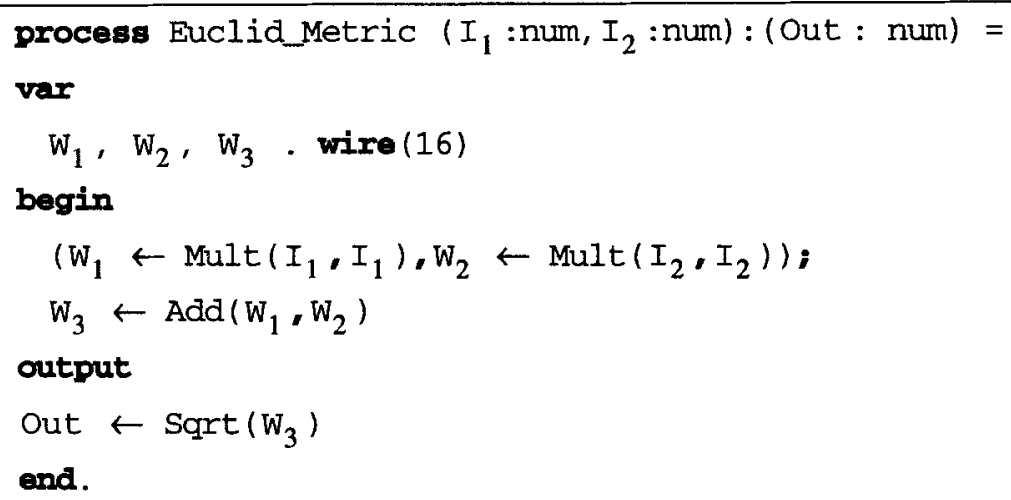

The corresponding hardware structure is given in figure 6-1. Note that the control signals have been deliberately omitted in the Add module. This optimization step is possible here, since a combinational module is used in a sequential composition and the overall behavior is not changed if the intermediate storage of the combinational results of Add is missing.

The base processes Mult, Add and Sqrt are verified as shown in section 5.2. The verification of the specification $\Phi$ of equation 6-1 is performed as shown in section 5.3.2 and section 5.3.3. Hence besides applying the respective simplification lemmata [4], only some internal lines have to be eliminated [16].

\section{Experimental Results}

Our approach has been successfully tested on several arithmetic circuits. Runtimes can be found in table 7-1. All runtimes have been achieved on a SUN SPARC 10 with $96 \mathrm{MB}$. Finding the loop invariants has turned out to be the main challenge in synthesizing the circuits. 


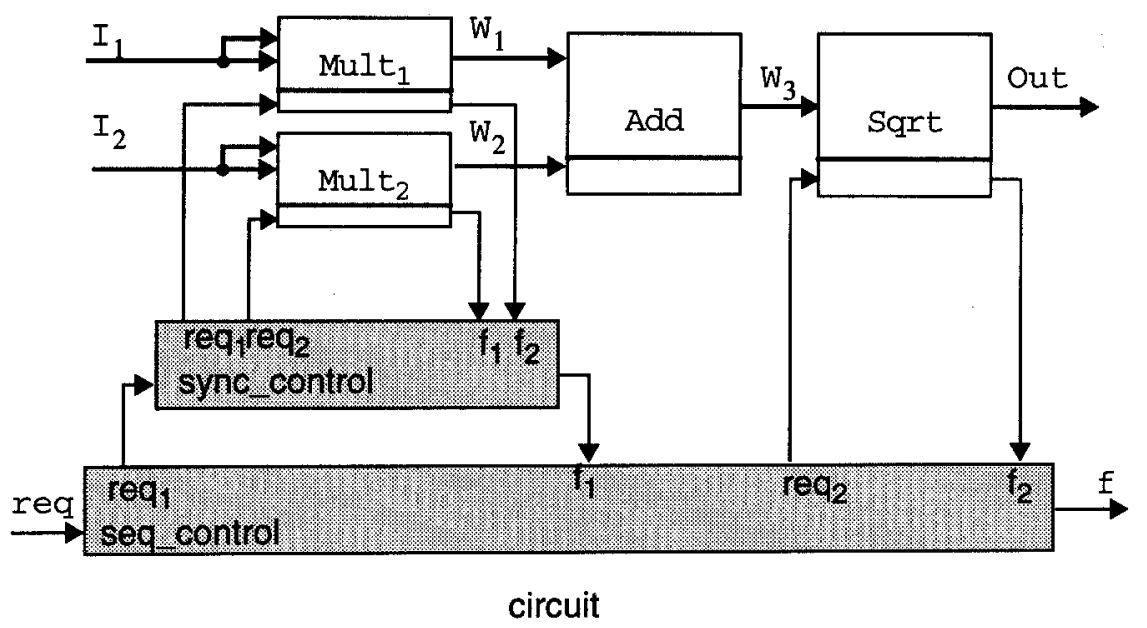

Fig. 6-1. Synthesized structure of the euclid metric

\begin{tabular}{|l|l|}
\hline \multicolumn{1}{|c|}{ Circuit Name } & \multicolumn{1}{c|}{ Time in seconds } \\
\hline VonNeumann 1 & 7.19 \\
\hline VonNeumann 2 & 17.33 \\
\hline IncDecAdder 1 & 6.22 \\
\hline IncDecAdder 2 & 15.81 \\
\hline Summation & 16.77 \\
\hline Signal Generator & 44.52 \\
\hline Greatest Cormon Divisor & 27.78 \\
\hline Russian Multiplier & 60.14 \\
\hline Divider & 73.60 \\
\hline SquareRoot & 66.85 \\
\hline
\end{tabular}

Table 7-1: Runtimes of different benchmark circuits

\section{Conclusions and Future Work}

In this paper, an approach to the formal synthesis of verification-friendly hardware structures has been presented. Having applied standard scheduling and allocation algorithms, known from high-level synthesis, an implementation description in a HDL-like language results. Its semantics is given directly as a composition of handshake circuits based on a synchronous handshake protocol. During the composition of these handshake circuits, a set of correctness theorems is derived which justifies the correctness of the specified circuit. Hence the correctness of scheduling and allocation, performed outside the formal framework, is checked. If during the specification phase, the designer's key ideas are provided via loop invariants, then all correctness proofs are done automatically, provided that a decidable domain like bitvectors have been used. 
In case of more complex data types, proof obligations on the data domain are derived automatically, which have then to be justified otherwise, e.g. by manual proofs. The approach is well suited for data driven applications, which are used in safety critical environments. For pure controller circuits, other approaches are better suited, e.g. by performing a post-verification based on model checking.

Using our approach, no time consuming post verification step is necessary to achieve correctness statements on the resulting circuits.

Obviously, the handshake module paradigm leads in some cases to suboptimal hardware solutions. We are currently investigating optimization strategies at higher abstraction levels like operator folding and low level techniques like multilevel logic minimization, FSM minimization and retiming to reduce hardware costs.

\section{References}

[1] M.C. McFarland, A.C. Parker, and R. Camposano. Tutorial on high-level synthesis. In 25th Design Automation Conference, pages 330-336, 1988.

[2] J.R. Burch, E.M. Clarke, K.L. McMillan, and D.L. Dill. Sequential circuit verification using symbolic model checking. 27rd ACM/IEEE Design Automation Conference. IEEE, 1990, pages 46-51.

[3] A. Gupta. Formal hardware verification methods: A survey. Journal of Formal Methods in System Design, pages 151-238, 1992.

[4] K. Schneider, R. Kumar, and T. Kropf. Automating verification by functional abstraction at the system level. In T. Melham and J. Camilleri, editors, International Workshop on Higher Order Logic Theorem Proving and its Applications, pages 391-406, Malta, September 1994. Lecture Notes on Computer Science No. 859, Springer.

[5] K. Schneider, R. Kumar, and T. Kropf. Modelling generic hardware structures by abstract datatypes. In L. Claesen and M. Gordon, editors, International Workshop on Higher Order Logic Theorem Proving and its Applications, pages 419429, Leuven, Belgium, September 1992. IFIP TC10/WG10.2, Elsevier Science Publishers.

[6] M. Mutz. Using the HOL theorem proving environment for proving the correctness of term rewriting rules reducing terms of sequential behavior. In K.G. Larsen and A. Skou. International Workshop on Computer Aided Verification, volume 575 of Lecture Notes in Computer Science. Springer, Aalborg, July 1991, pages 355-366.

[7] E. Mayger and M. P. Fourman. Integration of formal methods with system design. In A. Halaas and P.B. Denyer. International Conference on Very Large Scale Integration, pages 59-70, 1991. Edinburgh, North-Holland 
[8] M. Fujita and H. Fujisawa. Specification, verification and synthesis of control circuits with propositional temporal logic. J.A. Darringer and F.J. Rammig, editors. Computer Hardware Description Languages and their Applications, Washington, June 1989. IFIP WG 10.2, North-Holland, pages 265-279.

[9] S.M. Burns and A.J. Martin. Synthesis of self-timed circuits by program transformation. In G. Milne, editor, The Fusion of Hardware Design and Verification, pages 99-116, Glasgow, Scotland, July 1988. IFIP WG 10.2, NorthHolland.

[10] M.J.C. Gordon and T. Melham. Introduction to HOL: A Theorem Proving Environment for Higher Order Logic. Cambridge University Press, 1993.

[11] F.K. Hanna and N. Daeche. Specification and verification using high-order logic. In C.J. Koomen and T. Moto-oka, editors, Computer Hardware Description Languages, pages 418-433. Elsevier Science Publishers, North-Holland, 1985.

[12] E. A. Emerson. Temporal and Modal Logics, chapter 16, pages 996-1072. Elsevier Science Publishers, 1990.

[13] F. Kröger. Temporal Logic of Programs, volume 8 of EATCS Monographs on Theoretical Computer Science. Springer Verlag, 1987.

[14] K. Schneider, T. Kropf, and R. Kumar. Control-path oriented verification of sequential generic circuits with control and data path. In 4th European Design Automation Conference, pages 648-652, Paris, France, March 1994. IEEE Computer Society Press.

[15] T. Melham. Abstraction mechanisms for hardware verification. In G. Birtwistle and P.A. Subrahmanyam, editors, VLSI Specification, Verification and Synthesis, pages 267-291. Kluwer Academic Press, 1988.

[16] K. Schneider, T. Kropf, and R. Kumar. Why hardware verification needs more than model checking. In International Workshop on Higher Order Logic Theorem Proving and its Applications, Malta, 1994, Short Paper.

[17] R. Kumar, K. Schneider, and T. Kropf. Structuring and automating hardware proofs in a higher-order theorem-proving environment. International Journal of Formal System Design, pages 165-230, 1993. 